\title{
CONFECÇÃO DE MODELO NEUROANATÔMICO FUNCIONAL COMO ALTERNATIVA DE ENSINO E APRENDIZAGEM PARA A DISCIPLINA DE NEUROANATOMIA
}

\author{
CONFECCIÓN DE MODELO NEUROANATÓMICO FUNCIONAL COMO \\ ALTERNATIVA DE ENSEÑANZA Y APRENDIZAJE PARA LA ASIGNATURA DE \\ NEUROANATOMIA
}
FUNCTIONAL NEUROANATOMIC MODEL MANUFACTURE AS A TEACHING AND LEARNING ALTERNATIVE FOR NEUROANATOMY DISCIPLINE

\author{
Yslaíny ARAÚJO SILVA ${ }^{1}$ \\ Edivaldo Xavier da SILVA JÚNIOR ${ }^{2}$ \\ Bruno NERY SILVA ${ }^{1}$ \\ Gabriella PEREIRA RODRIGUES ${ }^{1}$ \\ Gabriella OLIVEIRA DE SOUSA ${ }^{1}$ \\ Wbinayara ALVES NOVAES ${ }^{1}$ \\ Ricardo Kenji SHIOSAKI ${ }^{3}$ \\ Tarcísio Fulgêncio ALVES DA SILVA ${ }^{4}$ \\ Paulo Adriano SCHWINGEL ${ }^{5}$
}

RESUMO: A neuroanatomia, disciplina que estuda as estruturas que compõem o Sistema Nervoso, é complexa, gerando dificuldades em seu processo de ensino-aprendizagem. Em seu ensino, aulas práticas integram as teóricas, utilizando cadáveres, materiais sintéticos e confeccionados. Estes últimos auxiliam a compreensão dos conteúdos, porém de forma segmentada. Buscar a integração funcional é uma importante ferramenta pedagógica em sua aprendizagem. Assim, o objetivo do estudo foi confeccionar um modelo neuroanatômico humano funcional alternativo para as aulas práticas da disciplina de neuroanatomia. $\mathrm{O}$ estudo foi uma pesquisa-ação, descritiva e exploratória, onde o modelo foi confeccionado por discentes monitores da disciplina, com material de baixo custo. Com

${ }^{1}$ Universidade de Pernambuco (UPE), Petrolina - PE - Brasil. Discentes do curso de Fisioterapia da UPE.

${ }^{2}$ Universidade Federal do Rio Grande do Sul (UFRGS), RS - Brasil. Docente Assistente de Anatomia Humana e Neuroanatomia da Universidade de Pernambuco Doutorando e mestre em Educação em Ciências. E-mail: edivaldo.junior@upe.br.

${ }^{3}$ Universidade de Pernambuco (UPE), Petrolina - PE - Brasil Docente do Programa de Pós-graduação Formação de Professores e Práticas Interdisciplinares (PPGFPPI) da Universidade de Pernambuco. Doutor em Ciências Biológicas pela Universidade Federal de Pernambuco (UFPE), PE - Brasil. E-mail: ricardo.shiosaki@upe.br.

${ }^{4}$ Universidade de Pernambuco (UPE), Petrolina - PE - Brasil. Docente do Programa de Pós-graduação Formação de Professores e Práticas Interdisciplinares (PPGFPPI) da Universidade de Pernambuco. Doutor em Ciências da Saúde pela Pontífica Universidade Católica do Paraná (PUCPR). E-mail: tarcisio.silva@upe.br.

${ }^{5}$ Universidade Federal do Rio Grande do Sul (UFRGS), RS - Brasil. Docente do Programa de Pós-graduação Educação em Ciências pela Universidade Federal do Rio Grande do Sul (UFRGS). Doutor em Medicina e Saúde pela Universidade Federal da Bahia (UFBA)., docente Adjunto da Universidade de Pernambuco. Email: paulo.schwingel@upe.br. 
isso, produziu-se um molde em folha de isopor do corpo humano, recoberto com emborrachado, bem como hemiestruturas direitas do Sistema Nervoso Central. Utilizaramse tintas coloridas que representassem a cor natural da maior parte das estruturas. Para a representação da funcionalidade do impulso nervoso, foram fixadas lâmpadas de led desde os membros até o córtex cerebral. Neurônios foram confeccionados e dispostos em todo o trajeto dos impulsos. Conclui-se que confeccionar modelos neuroanatômicos funcionais, com material de baixo custo, é viável e eficaz, o qual acredita-se contribuir com o ensinoaprendizagem da neuroanatomia.

PALAVRAS-CHAVE: Aprendizagem. Ensino. Neuroanatomia. Produção. Modelos.

RESUMEN: La neuroanatomía, asignatura que estudia las estructuras que componen el Sistema Nervioso, es compleja generando dificultades en su proceso de enseñanzaaprendizaje. En su enseñanza, clases prácticas integran las teóricas utilizando cadáveres, materiales sintéticos y confeccionados. Estos últimos auxilian la comprensión de los contenidos, pero de forma segmentada. La búsqueda de la integración funcional es una importante herramienta pedagógica en su aprendizaje. Así, el objetivo del estudio fue confeccionar un modelo neuroanatómico humano funcional alternativo para las clases prácticas de la asignatura de neuroanatomía. El estudio fue una investigación-acción, descriptiva y exploratoria, donde el modelo fue confeccionado por discentes monitores de la asignatura, con material de bajo costo. Con ello, se produjo un molde, en hoja de isopor del cuerpo humano, recubierto con una hoja de caucho, así como hemi-estructuras derecha del Sistema Nervioso Central. Se utilizaron tintas de colores que representaban el color natural de la mayoría de las estructuras. Para la representación de la funcionalidad del impulso nervioso, se fijaron lámparas de led desde los miembros hasta la corteza cerebral. Las neuronas fueron confeccionadas y dispuestas en todo el trayecto de los impulsos. Se concluye que confeccionar modelos neuroanatómicos funcionales, con material de bajo costo, es viable y eficaz, el cual se cree contribuir a la enseñanzaaprendizaje de la neuroanatomía.

PALAVRAS CLAVE: Aprendizaje. Enseñanza. Neuroanatomía. Producción. Modelos.

ABSTRACT: The neuroanatomy, discipline that studies the structures that make up the nervous system, is complex creating difficulties in its teaching-learning process. In its teaching, practical classes integrate the theoretical using cadavers, synthetic materials and manufactured. These latest assist the understanding of the contents, but in a segmented manner. Get functional integration is an important educational tool in its learning. The aim of the study was to manufacture an alternative functional human neuroanatomical model for the practical classes of neuroanatomy discipline. The study was an action research, descriptive and exploratory, where the model was made by students monitors of the discipline with low cost material. Thus, it was produced a template in a Styrofoam sheet of the human body overcoated with rubber and right hemistructures of the central nervous system. Have used color inks that represent the natural color of most structures. For the representation of the nerve impulse functionality, led lamps were fixed from the members to the cerebral cortex. Neurons were manufacture and arranged throughout the way of the impulses. We conclude that fabricate functional 
neuroanatomical models with low cost material, it is feasible and effective, which is believed to contribute to the teaching and learning of neuroanatomy.

KEYWORDS: Learning. Teaching. Neuroanatomy. Production. Models.

\section{Introdução}

A neuroanatomia é um ramo da anatomia humana responsável pelo estudo do sistema nervoso, central e periférico (D’ÂNGELO; FATTINI, 2007). O referido componente curricular aborda a anatomia morfológica e descritiva do sistema citado, através do estudo macro e microscópico de suas estruturas (MACHADO, 2014). Seus conteúdos são apresentados aos discentes de forma segmentada, porém sempre tentando esclarecer seus mecanismos através de diagramas, esquemas e imagens; contudo, não atendendo plenamente seus objetivos de aprendizagem. Complementando as aulas teóricas, as aulas práticas corroboram com o que foi estudado em sala de aula, permitindo a construção do conhecimento morfológico e, associadas às alternativas de ensino e aprendizagem, auxiliam na fixação de nomenclaturas das diversas estruturas que o compõe (LEITE; BORGES SILVA; RIBEIRO VAZ, 2005; ARAÚJO, 2008; FORNAZIERO et al., 2010). Além de trazer grandes desafios para os estudantes dos cursos de saúde, outras situações podem comprometer o dia a dia desta modalidade de aula, como a dificuldade de obtenção de corpos cadavéricos, imperícia técnica para manuseio e manutenção do acervo anatômico, como peças processadas sem nenhuma técnica de preservação de estruturas (RODRIGUES, 2005; MATTOS et al., 2008).

$\mathrm{Na}$ tentativa de suprir a escassez de material cadavérico, meios alternativos são utilizados constantemente no ensino da neuroanatomia, como livros, textos, atlas, modelos sintéticos, modelos anatômicos confeccionados, roteiros de aulas práticas, peças teatrais, uso de redes sociais, softwares, exames de imagem e laboratório digital (ALVES DA SILVA; LIMA E SILVA; SOUZA,2001; NOBESCHI, 2010; JAFFAR, 2012; JAFFAR, 2014; SILVA JÚNIOR et al., 2014a; SILVA JÚNIOR et al., 2014b; SHOEPE et al., 2015).

A confecção de modelos morfológicos como meio alternativo no ensino e aprendizagem da neuroanatomia já vem sendo aplicada em instituições de ensino superior, priorizando o desenvolvimento de projetos lúdicos (SILVA JÚNIOR et al., 2014a). A elaboração deste tipo de ferramenta pedagógica, além de despertar a curiosidade dos 
discentes, auxilia na aprendizagem dos conteúdos do componente citado (CABELLO; SINISCALCHI, 2009;SALING, 2007).

Aversi-Ferreira e colaboradores (2008) desenvolveram modelos que demonstravam vias e estruturas neurais, usando matéria-prima de baixo custo, como: madeira, tinta, barbantes, arames, entre outros. Os autores demonstraram que estas práticas, realizadas pelos próprios discentes, auxiliam em seu processo de aprendizado, contribuindo para que a vivência do conteúdo trabalhado seja mais concisa, além de minimizar os déficits de algumas universidades, tornando-se uma alternativa promissora a qualificar o aprendizado da anatomia humana e da neuroanatomia (SAILING et al., 2007; PEREIRA; PINTO, 2010; COLLIPAL; SILVA, 2011; CANEPPA et al., 2012; LIMA E SILVA; MACHADO; BIAZUSSI, 2012; CALAZANS, 2013; ANTONIA DA SILVA et al., 2014; ARAÚJO JÚNIOR et al., 2014; YAMMINE; VIOLATO, 2015).

Neste sentido, e com base na falta de recursos nas instituições de ensino superior, bem como a necessidade crescente na busca de novos paradigmas que aperfeiçoem e viabilizem a aprendizagem da neuroanatomia, o presente trabalho teve como objetivo confeccionar um modelo neuroanatômico humano funcional de baixo custo que seja útil em aulas práticas do referido componente curricular.

\section{Material e métodos}

O estudo foi do tipo pesquisa-ação, exploratória e descritiva. Foi desenvolvido pelos monitores da disciplina de neuroanatomia, bolsista e voluntários, no laboratório de Anatomia Humana da Universidade de Pernambuco - Campus Petrolina, no primeiro semestre de 2015. Utilizou-se material de baixo custo para a confecção do modelo, como: isopor, pincel, tinta acrilex, biscuit, massa de modelar, cola quente, "pisca-pisca" natalino, cano de pvc, retalhos de pano, emborrachado e cartolina.

Em reunião do grupo - monitores, colaboradores e orientador - decidiu-se que seria confeccionado um molde do corpo humano e nele afixado e reproduzidas as estruturas do sistema nervoso, com o foco na funcionalidade na condução de seus impulsos, desde a extremidade corpórea até a sua interpretação no córtex cerebral e, consequentemente, o processo de resposta por meio da utilização de lâmpadas de led.

Para a confecção, empregou-se os princípios da pesquisa-ação, a qual os participantes e o pesquisador estão envolvidos em uma mesma ação tendo por objetivo 
transformar a situação inicial em uma situação desejada (TRIPP, 2005). Com isso, e com a habilidade dos envolvidos, utilizando como base teórica o livro de Machado (2014), representou-se as estruturas fisiológicas condutoras dos impulsos nervosos, a fim de permitir uma visualização de todo o seu trajeto.

\section{Resultados e discussão}

Foi produzido um molde do corpo humano em folha de isopor recoberto com emborrachado, bem como um do hemisfério cerebral direito, do hemisfério cerebelar direito e um do hemi-tronco encefálico direito. Em seguida, foram misturadas tintas coloridas que representassem, o mais próximo possível, a cor natural da maior parte das referidas estruturas. Para a funcionalidade do impulso nervoso, fixou-se, com cola quente, lâmpadas de led desde os membros superiores e inferiores até a região do córtex cerebral. Os neurônios, do tipo multipolar, foram confeccionados com biscuit, em cores azul e vermelha, representando os neurônios aferentes e eferentes, respectivamente, e dispostos em todo o trajeto destes impulsos.

A primeira etapa da confecção consistiu na produção do molde do corpo humano, feito com isopor (Figura 1). Em seguida, esse molde foi coberto com emborrachado e algumas regiões foram pintadas com tinta de tecido. O cérebro e o cerebelo foram esculpidos em uma folha de isopor, baseando-se no livro atlas Sobotta (2013) e em seguida foram pintadas (Figura 2). Os sulcos e giros telencefálicos foram esculpidos com lixa de parede, dando o aspecto encontrado em peças neuroanatômicas naturais.

Figura 1: Cérebro e cerebelo esculpidos em isopor.

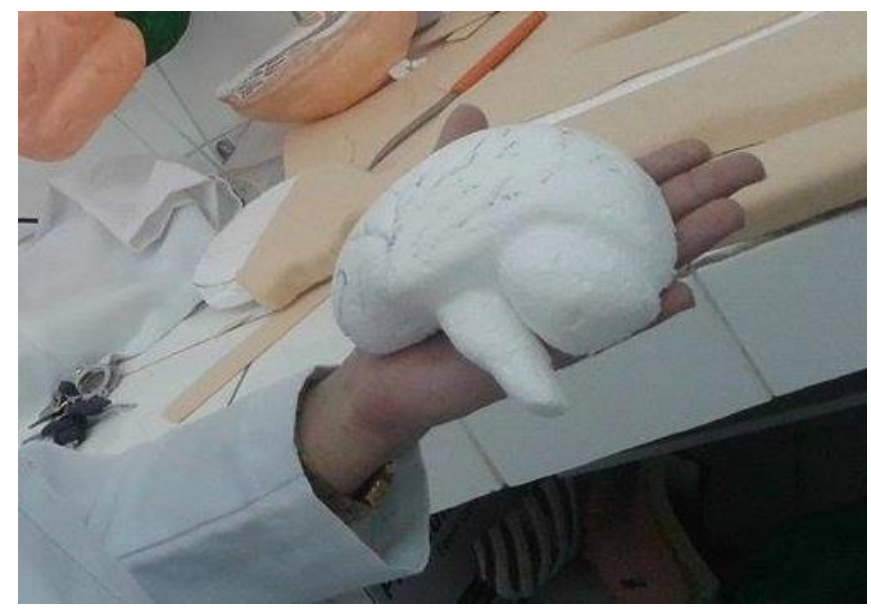

Fonte: Acervo dos próprios atores. 
Figura 2: Molde do corpo humano, recoberto com emborrachado.

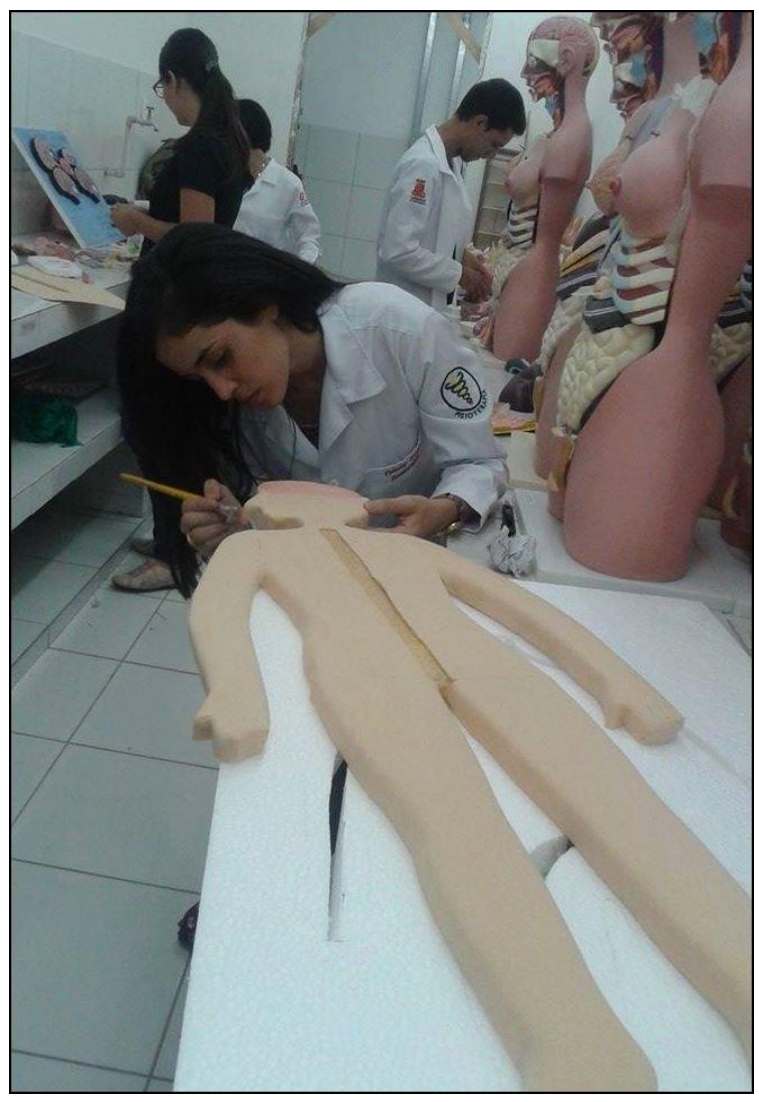

Fonte: Acervo dos próprios autores

Concluída a elaboração do molde, seguiu-se para a instalação da parte elétrica do modelo funcional didático, ao qual adicionou-se fitas de lâmpadas led representando todo o caminho do impulso nervoso, desde as extremidades apendiculares, superior e inferior, até a região cortical cerebral. Desta forma, foram representadas as sinapses ocorridas entre os neurônios, desde a captação do impulso nervoso até o local de sua interpretação, sendo distribuídas nas regiões cerebrais correspondentes a cada impulso.

Como estruturas pertencentes a todo esse trajeto envolvido, a medula espinhal e os nervos espinhais foram confeccionados e anexados ao molde, correspondendo a sua localização no corpo humano. Para a confecção da medula utilizou-se biscuit, e, para os nervos periféricos, barbantes com comprimentos semelhantes aos naturais, totalizando os 31 pares de nervos espinhais presentes no corpo humano. Os neurônios confeccionados foram sobrepostos às luzes de led, enfatizando os sentidos aferentes e eferentes através das cores em que foram produzidos. 
Confeccionado o modelo (Figuras 3 e 4), este foi utilizado em aulas da disciplina de neuroanatomia, visitas técnicas e oficinas realizadas no Laboratório de Anatomia Humana da Universidade de Pernambuco, Campus Petrolina.

Figura 3: Modelo Neuroanatômico funcional confeccionado.

Fonte: Acervo dos próprios autores

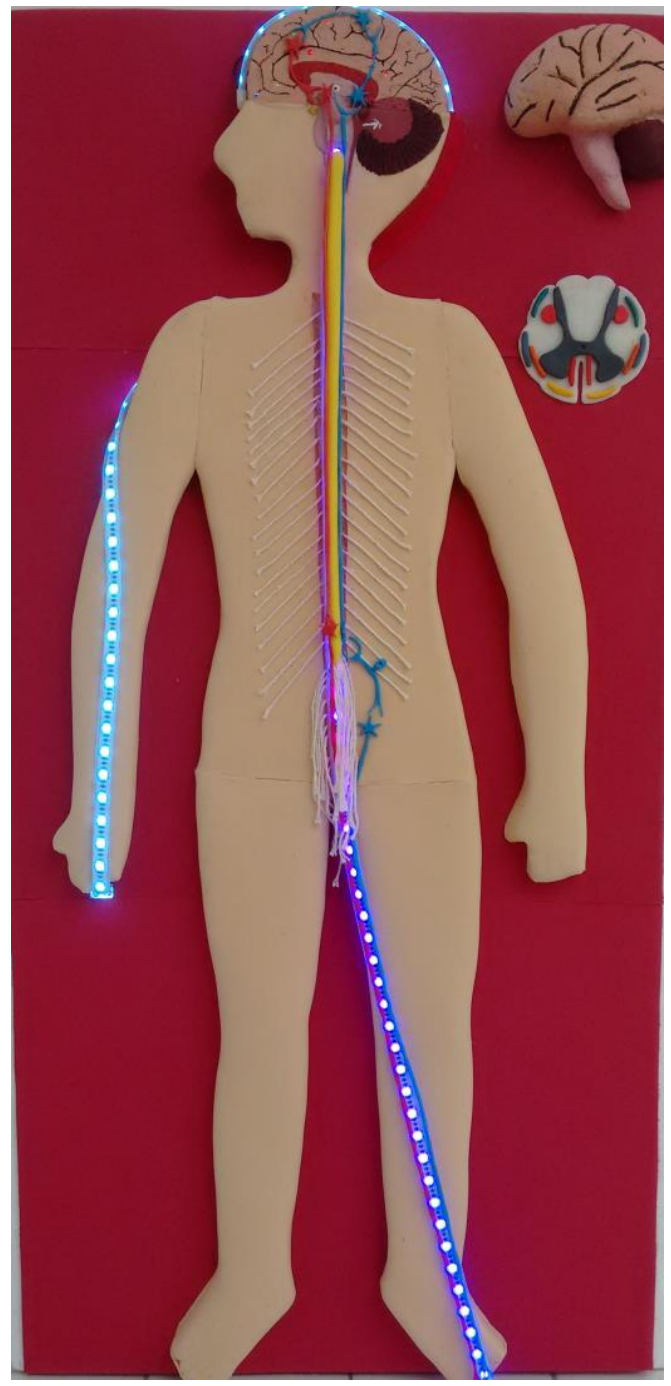


Figura 4: Modelo Neuroanatômico funcional confeccionado.

Fonte: Acervo dos próprios autores

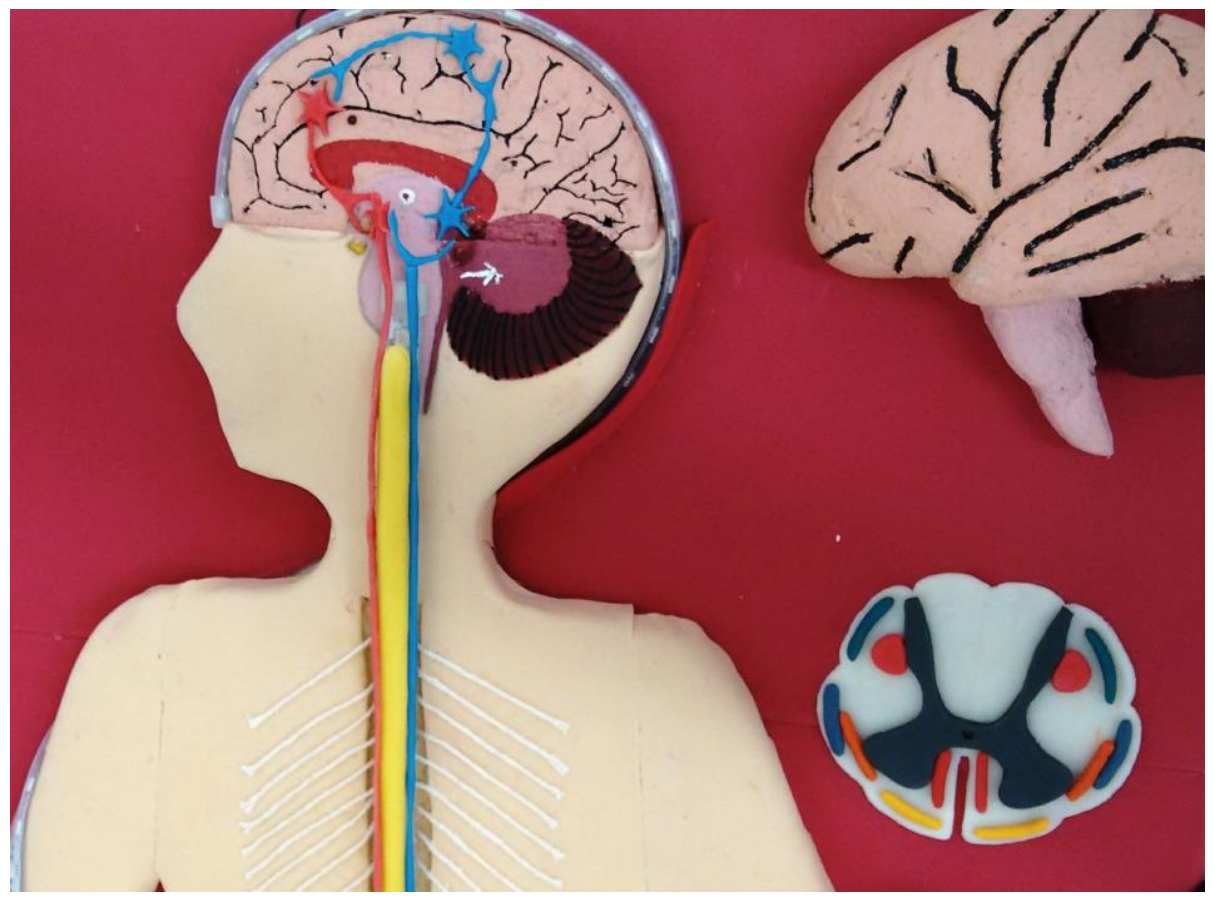

A modelagem surge como uma alternativa que pode ser utilizada na produção de cópias a partir de moldes anatômicos com foco nas diversas estruturas do corpo humano, podendo ser executada de diferentes maneiras (DUSO et al., 2013). Przybysz e Scolin (2008) retratam o uso dessa técnica na produção de moldes anatômicos das diversas regiões da coluna vertebral a partir de borracha de silicone e resina acrílica cristal. O objetivo dessas produções era suprir a falta de material cadavérico utilizado no ensino da anatomia humana e neuroanatomia. Semelhante ao objetivo dos autores, o presente estudo teve como propósito a obtenção de material para a vivência nas aulas práticas de Neuroanatomia, adquirindo modelo neuroanatômico funcional para o acervo do Laboratório de Anatomia Humana da Universidade de Pernambuco, Campus Petrolina.

Seguindo as mesmas vertentes, Blanco e colaboradores (2012) retratam a produção de replicas ósseas com resina de poliéster como uma alternativa didática na aprendizagem da anatomia. No referido estudo conseguiu-se reproduzir as peças de forma fidedigna, ou seja, as réplicas dos ossos do membro inferior foram representadas com riqueza de detalhes, incluindo os principais acidentes anatômicos. Desta forma, as confecções tornamse uma alternativa que possibilita o fornecimento de material de qualidade e resistência a ser utilizado no ensino e aprendizagem da referida disciplina. 
Vale ressaltar que as produções objetivam estimular os discentes a praticar as diversas técnicas de confecção de modelos para que estes adquiram conhecimentos, habilidades, experiências e criatividade, as quais poderão ser úteis em suas futuras carreiras profissionais.

Segundo Saling e colaboradores (2007), a produção de modelos anatômicos e maquetes humanas de qualidade é possível de ser realizada com custos relativamente baixos, como é o caso do uso da massa de biscuit, corroborando com Silva Júnior (2015). Segundo os autores, estes são didáticos e estimulam os alunos, que apresentam-se interessados em manipular os objetos confeccionados e aprofundar seus conhecimentos em relação aos assuntos abordados, permitindo que estes sejam agentes promotores do seu próprio processo de aprendizagem. Esse tipo de material facilita a modelagem das estruturas e as tornam acessíveis, como pode ser corroborado no presente artigo. Além disso, o uso do biscuit na preparação de estruturas confeccionadas por discentes incentivam e qualificam a aprendizagem, favorecendo e fornecendo subsídio aos docentes que, muitas vezes, acham-se limitados pela falta de peças anatômicas que representem, na prática, conteúdos estudados na teoria.

Segundo Silva Júnior e colaboradores (2014a), a elaboração de modelos anatômicos e a sua utilização nas aulas práticas em laboratórios de anatomia facilitam a aquisição do conhecimento pelos alunos, estimulando o seu interesse e participação. Ainda de acordo com esses autores, os modelos podem ser confeccionados com qualidade e com material de baixo custo, sendo uma alternativa eficaz diante das adversidades relacionadas à aquisição de peças cadavéricas. Corroborando com esses pesquisadores, o material de baixo custo utilizado na construção do presente modelo funcional demonstrou-se acessível, viável e de fácil manipulação, sendo reiterado, assim, o seu uso em desenvolvimento de práticas didáticas em sala de aula ou em laboratórios de ensino em anatomia humana.

Araújo Júnior e colaboradores (2014), enfatizam que o processo de confecção é uma forma de participação ativa na aprendizagem, estando envolvidas as habilidades, os conhecimentos e a criatividade dos discentes. Com isso, a promoção da interação mútua entre o indivíduo e o objeto estudado é aspecto facilitador na apreensão do conteúdo. Um aspecto importante abordado pelos autores foi a contribuição deste tipo de processo para a formação da memória a longo prazo, de grande relevância, já que estes conhecimentos serão utilizados pelos futuros profissionais de saúde em toda a sua vivência acadêmica e profissional. 
Assim, o referido trabalho legitima o presente estudo, uma vez que constatou-se o envolvimento dos discentes monitores no processo de confecção, o qual os aperfeiçoou em seus conhecimentos na disciplina de neuroanatomia, permitindo-lhes confeccionar as estruturas de forma viável e fidedigna, tornando-os agentes ativos em seu processo de aprendizado.

López e colaboradores (2011) relatam a utilização de modelos anatômicos em algumas Universidades do Chile com a finalidade de facilitar a aprendizagem da anatomia frente à dificuldade do estudo em cadáveres humanos como consequência da sua difícil aquisição. Na visão dos estudantes questionados, pode-se constatar a alta valorização da utilização dos modelos, atrelando seu uso à aprendizagem, habilidades, valores e atitudes, como trabalho em equipe, responsabilidade, respeito e criatividade.

Complementando os autores acima, Collipal e Silva (2011) constataram que este recurso cumpre o objetivo de ser uma alternativa para a aprendizagem da Anatomia Humana. Ainda conforme os autores, faz-se essencial destacar a importância da utilização conjunta de recursos cadavéricos e modelos anatômicos durante as aulas práticas da disciplina. Os modelos alternativos, não cadavéricos, assim como outros recursos, a exemplo da multimídia e de softwares, são utilizados como um apoio aos alunos em sua apreensão do conteúdo essencial em seu processo de graduação. Diante disto, o produto do presente trabalho apresenta-se como mais uma alternativa na busca da compreensão dos extensos assuntos da neuroanatomia.

Estudos realizados por Narváez-Hernández e Murilo-Rábago (2014) demonstraram que os modelos anatômicos prevaleciam como uma ferramenta útil em relação a vídeos, diagramas, desenhos, exposições e perguntas exploratórias. Sendo assim, levando-se em conta as limitações que se tem devido aos orçamentos atribuídos às universidades e as diversas dificuldades referentes à aquisição de cadáveres humanos, os modelos apresentam-se como ferramenta com grande potencial didático.

Além de modelos anatômicos, estudos voltados para o ensino-aprendizagem da neuroanatomia evidenciam a utilização, também, de mapas conceituais, aprendizagem baseada em problemas, neuroanatomia computacional, ambientes virtuais e técnicas como a estereoscopia, com o objetivo de enriquecer o seu aprendizado (MENESES, 2002; CHAO GONZÁLEZ, 2006; AYALA-PIMENTEL; DÍAZ-PÉREZ; OROZCO-VARGAS, 2009; BLANCO, 2010). 
Como pode-se observar, há um acervo satisfatório na literatura referente à utilização de modelos anatômicos no ensino-aprendizagem da disciplina de anatomia humana, englobando diversos métodos de confecção e diferentes tipos de materiais a serem utilizados, assim como estudos direcionados a outras formas alternativas que auxiliam e apoiam o estudo da anatomia. Entretanto, após análise dos achados literários, não há estudos que enfatizem a confecção e utilização de modelos neuroanatômicos funcionais, sendo esta uma problemática a ser abordada e sanada em futuros estudos nessa área, mostrando-se como uma proposta inovadora que contribuirá no ensino e aprendizagem da neuroanatomia.

\section{Considerações finais}

Conclui-se que confeccionar modelo funcional neuroanatômico apresenta-se como uma ferramenta alternativa de fácil manipulação, a qual acredita-se contribuir com o ensino-aprendizagem das estruturas neuroanatômicas microscópicas e macroscópicas, já que demonstram a relação entre elas no interior do corpo humano. Quanto à confecção com material de baixo custo, frente à aquisição de modelos fabricados, reitera-se que é viável, tornando-se uma ferramenta pedagógica diante das dificuldades na compreensão da neuroanatomia. Além disso, baseando-se na literatura, a proposta apresenta-se como inovadora, diante dos diversos métodos utilizados no processo de ensino-aprendizagem do referido componente curricular nas Instituições de Ensino Superior.

AGRADECIMENTOS: Ao Programa de Fortalecimento Acadêmico (PFA) da Universidade de Pernambuco (UPE) pelo financiamento, através de bolsa de monitoria, e à toda a equipe do Laboratório de Estudo e Pesquisa em Anatomia Humana (LABEPAH) da Universidade de Pernambuco, Campus Petrolina.

\section{REFERÊNCIAS}

ALVES DA SILVA, R.; LIMA E SILVA, M.; SOUZA, R. O ensino da Anatomia através das artes cênicas. Arquivos da Apadec, v. 5, n. 1, p. 9-14, 2001.

ANTONIA DA SILVA A. et al. O uso do biscuit como ferramenta complementar ao ensino de anatomia humana: um relato de extensão universitária. Revista Ciência em Extensão. v. 10, n. 2, p.47-54, 2014. 
ARAÚJO, A. O. O uso do tempo e das práticas epistêmicas em aulas práticas de química. 2008. 144f. Dissertação (Mestrado em Educação). Faculdade de Educação Universidade Federal de Minas Gerais, Belo Horizonte - MG, 2008. Disponível em: $<$ http://www.bibliotecadigital.ufmg.br/dspace/bitstream/handle/1843/FAEC85BKEK/disserta_o_ang_lica_oliveira_de_ara_jo.pdf?sequence=1 >. Acesso em $30 \mathrm{mar}$. 2016.

ARAÚJO JÚNIOR, J. P. et al. Desafio anatômico: uma metodologia capaz de auxiliar no aprendizado de anatomia humana. Revista da Faculdade de Medicina de Ribeirão Preto e do Hospital das Clínicas da FMRP, v. 47, n. 1, p. 62-68, 2014.

AVERSI-FERREIRA, T. A. et al. Estudo de Neurofisiologia Associado com Modelos Tridimensionais Construídos Durante o Aprendizado. Bioscience Journal, v. 24, n. 1, p. 98-103, 2008.

AYALA-PIMENTEL, J. O.; DÍAZ-PÉREZ, J. A.; OROZCO-VARGAS, L. C. Eficacia de la utilización de estilos de aprendizaje en conjunto con mapas conceptuales y aprendizaje basado en la resolución de problemas para el aprendizaje de neuroanatomía. Educación Médica, v. 12, n. 01, p. 25-31, 2009.

BLANCO, Y. Q. et al. Réplicas óseas en resina poliester como herramienta didáctica para el aprendizaje de anatomía. Revista U.D.C.A Actualidad \& Divulgación Científica, v. 15 , n. 2, p. 275-281, 2012.

BLANCO, Y. Q. Impacto del uso de entornos virtuales de aprendizaje para la enseñanza de neuroanatomía en estudiantes de medicina. Revista U.D.C.A. Actualidad \& Divulgación Científica, v. 13, n. 2, p. 15-22, 2010.

CABELLO, C. A. S.; SINISCALCHI, M. H. A contribuição e socialização dos conhecimentos da neuroanatomia no contexto da sala de aula, no processo de ensino e aprendizagem. Revista Ibero-americana de Educação. v. 1, n. 51, p. 01-06, 2009.

CALAZANS, N. C. O ensino e o aprendizado práticos da anatomia humana: uma revisão de literatura. 2013. 59f. Monografia de Conclusão de Curso de Graduação Faculdade de Medicina da Bahia, Universidade Federal da Bahia, Salvador, 2013.

CANEPPA, A. R. G. et al. Utilização de modelos didáticos no aprendizado de anatomia e fisiologia cardiovascular. Revista do Curso de Enfermagem, v. 01, n. 01, p. 01-07, 2012. Disponível em: http://www.santacruz.br/ojs/index.php/Revenf/article/view/1013/931. Acesso em: 02 jan. 2016.

CHAO GONZÁLEZ, S. et al. Nuevas herramientas para el desarrollo de la neuroanatomía computacional. Revista Cubana de Investigaciones Biomédicas, v. 25, n. 4, 2006. Disponível em: <http:// http://scielo.sld.cu/scielo.php?script=sci_arttext\&pid=S086403002006000400003>. Acesso em: 02 jan. 2016.

COLLIPAL, L. E.; SILVA, M. H. Estudio de la Anatomía en cadáver y modelos anatómicos. Impresión de los estudiantes. International Journal Morphological, v. 29, n. 4, p. 1181-1185, 2011. 
DANGELO, J. G.; FATTINI, C. A. Anatomia humana sistêmica e segmentar. 3. ed. Rio de Janeiro: Atheneu, 2007.

DUSO L. et al. Modelização: uma possibilidade didática no ensino de biologia. Revista Ensaio, v. 15, n. 02, p. 29-44, 2013.

FORNAZIERO, C. C. et al. O ensino da anatomia: integração do corpo humano e meio ambiente. Revista Brasileira de Educação Física, v. 34, n. 2, p. 290-297, 2010.

GRAY, H.; GOSS, C. M. Anatomia. 29 ed. Rio de Janeiro: Guanabara Koogan, 2012.

NARVÁEZ-HERNÁNDEZ, E.; MURILO-RÁBAGO, E. I. Herramientas de estudio utilizadas por alumnos de ciencias de la salud en la materia de Anatomía. Investigación en Educación Médica, v. 3, n. 12, p. 204-208, 2014.

JAFFAR, A. A. YouTube: an emerging tool in anatomy education. Anatomical Science Education, v. 5, n. 3, p. 158-164, 2012.

JAFFAR, A. A. Exploring the use of a facebook page in anatomy education. Anatomical Science Education, v. 7, n. 3, p. 199-208, 2014.

LEITE, A. C. S.; BORGES SILVA, P. A.; RIBEIRO VAZ, A. C. A importância das aulas práticas para alunos jovens e adultos: uma abordagem investigativa sobre a perspectiva dos alunos do PROEF II. Ensaio Pesquisa em Educação em Ciências, v. 07, n. 03, p. 01-16, 2005 .

LIMA e SILVA M. S.; MACHADO H. A.; BIAZUSSI H. M. Produção de material didático alternativo para aula prática de anatomia humana. VII CONNEPI, Congresso Norte e Nordeste de Pesquisa e Inovação, Anais... Palmas -Tocantins, 2012. Disponível em: <http://propi.ifto.edu.br/ocs/index.php/connepi/vii/paper/viewFile/4211/1560>. Acessado em: 02 jan. 2016.

LÓPEZ, F. B. et al. Valoración de la actividad de modelos anatómicos en el desarrollo de competencias en alumnos universitarios y su relación con estilos de aprendizaje, carrera y sexo. International Journal Morphological, v.29, n. 2, p.568-574, 2011.

MACHADO, A. B. M. Neuroanatomia funcional. 3 ed. São Paulo: Atheneu, 2014.

MATTOS, J. P. Dissection technique for the study of the cerebral sulci, gyriand ventricles. Arquivo de Neuropsiquiatria. v. 66, n. 2A, p.282-287, 2008.

MENESES, M. S. et al. Estereoscopia aplicada à Neuroanatomia. Estudo comparativo entre as técnicas de filtro de cores e de polarização. Arquivos de Neuro-Psiquiatria, v. 60, n. 3-B, p. 769-774, 2002.

NOBESCHI, L. Introdução ao estudo da Anatomia Humana. Instituto de Imagens em Saúde - CIMAS, 2010. Disponível em: <http://www.imagingonline.com.br/ biblioteca/Leandro_Nobeschi/introducao-ao-estudo-da-anatomia-humana.pdf $>$. Acesso em 04 mai. 2015. 
ORLANDO, T. C. et al. Planejamento, montagem e aplicação de modelos didáticos para abordagem de biologia celular e molecular no ensino médio por graduandos de ciências biológicas. Revista Brasileira de Ensino de Bioquímica e Biologia molécula, v. 07, n. 1, p. 1-17, 2009.

PEREIRA, I. P.; PINTO, K. S. O. Desenvolvimento de uma maquete para representação do sistema nervoso central - trato córtico espinhal. 2010. 39p. Monografia de Conclusão de Curso de Graduação - Universidade Federal do Paraná, 2010.

PRZYBYSZ, C.; SCOLIN, E. Técnica anatômica: confecção de modelos em resina a partir de vértebras humana. F@P Ciencia, v. 2, n. 10, p. 1-12, 2008. Disponível em: $\langle$ http://www.fap.com.br/fapciencia/002/edicao_2008/010.pdf $>$. Acessado em: 15 set. 2015.

RODRIGUES, H. Técnicas Anatômicas. 3 ed. Vitória: Própria, 2005.

SALING, S. C. et al. Modelos didáticos anatômicos: um recurso a ser explorado. In: V Encontro Internacional de Produção Científica do Cesumar - EPCC, Maringá. Anais..., Paraná: V EPCC, 2007. Disponível em: <http://www.cesumar.br/prppge/pesquisa/ epcc2007/anais/simoni_cristina_saling.pdf>. Acessado: 23 de julho de 2015.

SHOEPE, T. C. et al. The ATLAS project: the effects of a constructionist digital laboratory Project on undergraduate laboratory performance. Anatomical Science Education, v. 08, n. 01, p. 12-20, 2015.

SILVA JÚNIOR, E. X. Avaliação do uso de modelos anatômicos alternativos para o ensino-aprendizagem da anatomia humana para alunos do ensino fundamental de uma Escola Pública da cidade de Petrolina, PE. 2015. 98f. Dissertação (Mestrado em Educação em Ciências: Química da Vida e Saúde). Universidade Federal do Rio Grande do Sul, Porto Alegre - RS, 2015. Disponível em:

$\langle$ http://www.lume.ufrgs.br/bitstream/handle/10183/132326/000982957.pdf?sequence=1 $>$. Acessado em: 05 abr. 2016.

SILVA JÚNIOR, E. X. et al. Elaboração de modelos anatômicos alternativos para o ensino-aprendizagem da disciplina de neuroanatomia humana, a partir de material de baixo custo. In CONEDU, 1, 2014a, Campina Grande. Anais... Paraíba: Realize, 2014. p. 1-5. Disponível em:

<http://www.editorarealize.com.br/revistas/conedu/trabalhos/Modalidade_1datahora_14_ 07_2014_19_51_54_idinscrito_3160_eec18973fabcaf6b30f5b526540557cc.pdf>.

Acessado em: 15 set. 2015.

SILVA JÚNIOR, E. X. et al. Elaboração de roteiros para o ensino-aprendizagem nas aulas práticas das disciplinas de anatomia humana e neuroanatomia. In CONEDU, 1. 2014b, Campina Grande. Anais... Paraíba: Realize, 2014. p. 1-5. Disponível em: < http://www.editorarealize.com.br/revistas/conedu/trabalhos/Modalidade_1datahora_15_0 7_2014_22_59_18_idinscrito_3160_6ad18d13d81622ec0227b39630b0b40a.pdf>. Acessado em 15 set. 2015. 
SOBOTTA, J.; PUTZ, R.; PABST, R. D. Sobotta atlas de anatomia humana. 3 v. 23 ed. Rio de Janeiro: Guanabara Koogan, 2013.

TRIPP, D. Pesquisa-ação: uma introdução metodológica. Educação e pesquisa, v. 31, n. 3, p. 443-466, 2005.

YAMMINE, K.; VIOLATO, C. The effectiveness of physical models in teaching anatomy: a meta-analysis of comparative studies. Advances in Health Sciences Education, p. 1-13, 2015. Disponível em: <https://link.springer.com/article/10.1007/s10459-015-9644-7>.

Acessado em: 02 jan. 2016.

\section{Como referenciar este artigo}

SILVA, Yslaíny Araújo et al. Confecção de modelo neuroanatômico funcional como alternativa de ensino e aprendizagem para a disciplina de neuroanatomia Revista IberoAmericana de Estudos em Educação, Araraquara, v. 12, n. 3, p. 1674-1688, jul-set/2017. Disponível em: <http://dx.doi.org/10.21723/riaee.v12.n.3.2017.8502>. E-ISSN: 19825587.

Submetido em: 05/04/2016

Aprovação final em: 10/07/2017 